\title{
Data Spider: A Resilient Mobile Basestation Protocol for Efficient Data Collection in Wireless Sensor Networks
}

\author{
Onur Soysal, Murat Demirbas \\ Computer Science \& Engineering Dept., \\ University at Buffalo, SUNY \\ Email: \{osoysal,demirbas\}@ cse.buffalo.edu
}

\begin{abstract}
Traditional deployments of wireless sensor networks (WSNs) rely on static basestations to collect data. For applications with highly spatio-temporal and dynamic data generation, such as tracking and detection applications, static basestations suffer from communication bottlenecks and long routes, which cause reliability and lifetime to plummet.

To address this problem, we propose a holistic solution where the synergy of the WSN and the mobile basestation improves the reliability and lifetime of data collection. The WSN component of our solution is a very lightweight dynamic routing tree maintenance protocol which tracks the location of the basestation to provide an always connected network. Our basestation algorithm complements the dynamic tree reconfiguration protocol by trailing towards the data generation, and hence, reducing the number of hops data needs to travel to the basestation. While both protocols are very simple and lightweight, combined they lead to significant improvements in the reliability and lifetime of data collection. We provide an analytical discussion of our solution together with detailed discrete event simulations.
\end{abstract}

\section{INTRODUCTION}

The objective for deploying a wireless sensor network (WSN) is to collect data from an area for some time interval. Traditionally, a static basestation (SB) is deployed with the WSN, and the WSN nodes relay data over multihops towards the SB, which stores/uploads the data for processing. In order to improve the efficiency (which determines the lifetime) and reliability (which determines the quality) of data collection, most of the research focus on the relay nodes. Numerous schemes have been proposed for coordinating sleep-wake-up, aggregation techniques, and routing structures of the relay nodes. On the other hand, relatively little attention is given to improve/change the basestation model, and investigate holistic solutions to the data collection problem.

The traditional SB model has several handicaps. A primary problem is that the SB constitutes a hot spot for the system. Since the nodes closer to SB is always employed in relaying the entire traffic, these nodes deplete their batteries quickly, putting a cap on the lifetime of the deployment. Another major problem is due to the spatio-temporal nature of the data generation. In several WSN deployments, including environmental monitoring [1], habitat monitoring [2], and especially surveillance systems [3], [4], it has been observed that the phenomena of interest are local both in time and space. Fixing the location of the basestation ignores the nature of the data generation and results in long multihop paths for relaying, which leads to a lot of collisions and data losses.
In order to address the drawbacks of the SB model, several work proposed to deploy a mobile basestation (MB) for data collection. The classical "data mule" work [5] proposed to exploit random movement of MBs to opportunistically collect data from a WSN. Here, the nodes buffer their data and upload only when the MB arrives within direct communication. Although this approach eliminates multihop data relaying, the tradeoff is the very high latency, which makes the approach unsuitable for real-time monitoring applications. To fix the latency problem, the mobile element scheduling (MES) work [6] considered the controlled mobility of the $\mathrm{MB}$ and studied the problem of planning a path for the MB to visit the nodes before their buffers overflow (which turned out to be an NP-complete problem [6], [7]). MES work, however, assumes that the data-rates in the WSN are known and fixed (constant after initialization), which is very limiting for monitoring applications. Controlled sink mobility in [8] reduces latencies significantly through maintenance of routes to sink location from all nodes. Optimal solution for this model requires preprocessing similar to MES, but authors also propose a greedy alternative. Since reactive sink mobility requires flooding of whole network, this study assumes the sink stays for relatively long durations on small number of predefined sink locations. This limits its ability to address dynamic data generation in an agile manner.

In our previous work, we presented a holistic, networked controlled MB algorithm, "data salmon" [9]. Data salmon constructs a backbone spanning tree over the WSN, and constrains both the data relaying and the MB movements to occur on this tree. The MB strategy is to greedily relocate to the subtree where most of the traffic originates. 1 In return when the MB moves along one edge of the tree, it updates the direction of the edge to point to its new location to ensure that the root of the backbone tree is switched to be at the new location of MB. Hence tracking of the MB is achieved with minimum cost.

While achieving low cost tracking and reducing the average weighted relay distance of data, the data salmon also has some shortcomings. The hotspot problem is still unresolved: since data salmon uses a static backbone tree, the center of the static backbone tree still relays a significant amount of traffic and is

\footnotetext{
${ }^{1}$ We showed that this greedy strategy is optimal, under the constraints of limiting all the data relaying to occur on the static backbone tree.
} 
a potential hotspot. Moreover, a static backbone tree implies that a message-loss during the handoff of MB from one node on the tree to the next leads to a permanent partitioning. 2

Our contributions. To address the shortcomings of data salmon, we introduce data spider. Data spider relaxes the static backbone tree requirement in the data salmon, and maintains a dynamic tree for the data collection. This tree is updated locally and efficiently by the movements of the MB. The visual imagery is that of a spider (corresponding to the MB) re-weaving/repairing its web (corresponding to the tree) as it moves. To complete the feedback loop, the spider relies on its web to detect interesting phenomena (data generation) to follow.

Data spider fixes the hotspot problem of data salmon. Our simulation results show that data spider extends the lifetime of the deployment by several folds over the data salmon. Due to its dynamicaly reconfigured tree, data spider is also resilient. Data spider does not depend on a static backbone tree for routing, so faults/message-losses are not as catastrophic in data spider as in data salmon.

The dynamic tree reconfiguration protocol in data spider is of interest on its own accord. The philosophy here is to update the tree at where it counts, where the most recent action is. So instead of trying to maintain a distance-sensitive tree for the entire network (which is clearly a non-local task), we maintain a temporally-sensitive tree by reconfiguring the tree only at the immediate locality of the MB. As such the maintenance cost of the tree is very low. Yet this does not lead to long and inefficient paths for data relaying to MB: since the MB follows the data generation closely (thanks to the MB's trailflow algorithm), the effective length of paths is only a couple of hops. We show this in our simulation results.

To investigate the requirements for proper handoffs by the $\mathrm{MB}$, we formulate the handoff connectivity property. Handoff connectivity, intuitively, captures the notion of having no holes in the network. We note that our data spider does not require the handoff connectivity in practice. We devise a simple yet very effective algorithm - trail-flow algorithm-for the MB, that avoids bad handoffs by routing the MB around the holes. In the trail-flow algorithm, the MB follows the edges where the most data is flowing to itself, in contrast to a follow-source approach of going to the source of the data directly. As we show in our simulation results, follow-source leads to several incorrect handoffs whereas trail-flow still functions correctly in the same density/network.

We give simulation results to investigate the scalability and efficiency of data spider, and compare it with data salmon and the SB approach. Our simulator uses realistic lossy channel models and provides a high-fidelity energy calculation by using BMAC [10] as the model for the MAC layer communications. 3 Our simulation results show that data spider

\footnotetext{
${ }^{2}$ Requiring acknowledgment messages alleviates the problem, but also increases the overhead of the protocol significantly.

${ }^{3}$ Since our simulator is parametrized extensively it is suitable for modeling and investigating other MB algorithms quickly. Our simulator is available at http://www.cse.buffalo.edu/ubicomp/dataSpider/
}

outperforms data salmon and the SB approaches consistently, and leads to significant improvements in the reliability and lifetime of data collection.

We focus on one mobile region of interest (ROI) and on one MB. When multiple ROIs are present with only a single MB to follow them the performance of data spider is reduced. However, we show that data spider extends readily to allow several MBs to share the same network. There is no need to change the routing in WSN or heuristics of the MB. We also provide simulation results using multiple MBs to collect data from multiple ROIs.

Outline of the rest of the paper. Our data spider consists of two submodules: a dynamic tree reconfiguration protocol, and an algorithm for MB relocation. We present our dynamic tree reconfiguration protocol, DTR (read detour), and discuss the handoff connectivity requirements for DTR in Section III In Section III, we present our basestation algorithm for relocation. We give simulation results in Section IV on the scalability and efficiency of our data spider, comparing and contrasting it with a static basestation and data salmon scheme.

\section{Dynamic Tree Reconfiguration}

In this section, we first present the DTR algorithm. We give the correctness proof of DTR in Section $\Pi-\mathrm{B}$ and present the handoff connectivity requirements for DTR in Section II-C Finally, we present extensions to the basic DTR in Section II-D

\section{A. DTR Algorithm}

To maintain always-on connectivity to the $\mathrm{MB}$, the network should continuously track it and update the existing routing paths to point to its new location. Trying to maintain a distance-sensitive tracking structure (e.g., maintaining a shortest path tree rooted at the MB) would be beneficial since it would reduce the number of hops data need to be relayed towards the MB. However, this is inherently a non-local and costly task as it requires frequent multihop broadcasts.

Since energy-efficiency is of utmost importance for improving the lifetime of data collection, in our dynamic tree configuration protocol, DTR, we take an alternative approach. To keep the maintenance cost of the tree very low, we confine DTR to reconfigure the tree only at the immediate locality (singlehop) of the MB. To ensure that DTR does not beget long and inefficient paths for data relaying to $\mathrm{MB}$, we rely on the MB algorithm. In our simulation results in Section IV-B we show that since the MB's trail-flow algorithm follows the data generation closely, the effective length of data relaying paths is only a couple of hops.

DTR starts with a spanning tree rooted at the MB. This could be established by constructing an initial tree using flooding and keeping the MB static. The root node of this initial tree is called the anchor node, which is also the closest node to the MB. As it relocates in the network, MB chooses the anchor node to be the closest node to itself and makes periodic broadcasts to declare the anchor node to all nodes in its singlehop range. Nodes that receive the anchor broadcast 
update their parents (next pointers) to point to the new anchor node. At any time there is a unique anchor node in the network, which is maintained to be the closest node to the MB.

We present DTR in Algorithm 1. Only the nodes that receive the anchor broadcast execute an action and update their next pointers. The anchor broadcasts are local to the singlehop of the MB and they are not relayed to multiple hops. Figure 1 depicts an example of DTR execution.

Dynamic convoy tree work [11] adresses a relevant dynamic tree reconfiguration problem in the context of target tracking. Dynamic convoy tree maintains a monitoring tree to cover a mobile ROI. The root of this monitoring tree controls expansion and contraction of the tree and when needed decides on the relocation of the root to another node based on the information it collects from the entire tree. Our advantage in DTR is the cooperation of the MB for relocating the root of the tree to an optimal location using local singlehop updates, whereas the convoy tree needs to deal with the tree reconfiguration problem by using multihop update messages.

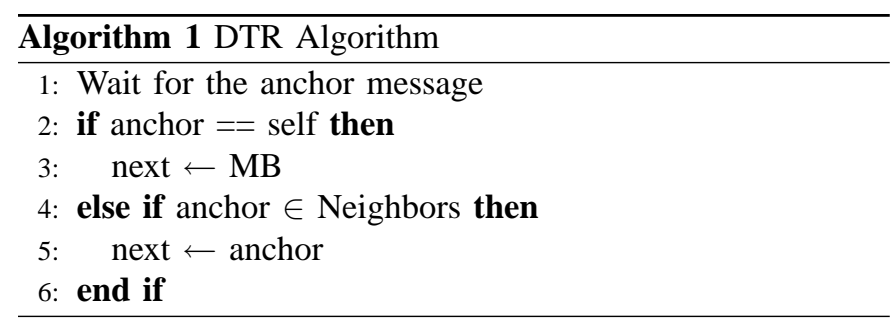

\section{B. Correctness}

For clarity of explanation we use the following definitions:

Definition 1: (Handoff): the operation with which the MB changes the anchor node from one node to another.

Definition 2: (Routing connected): a network where the next links of nodes form a spanning routing tree rooted at MB.

Definition 3: (Proper handoff) A handoff is proper when:

1) Both the old and new anchor nodes receive the MB's anchor broadcast

2) Both the old and new anchor nodes can reliably communicate with each other

Theorem 1: If all handoffs are proper, an iteration of Algorithm 1 starting from a routing connected network always results in another routing connected network.

Proof: Consider tree reconfiguration on a graph $G=$ $(V, E)$ where $u, v \in V$ correspond to the nodes and $e=$ $(u, v) \in E$ correspond to the reliable communication links between the nodes. We use $r$ to denote the old anchor and $r^{\prime}$ to denote the new anchor. In the base case, when there is no handoff, $r^{\prime}=r$, and the theorem holds vacuously. We next consider the case where $r^{\prime} \neq r$.

The iteration of Algorithm 1 entails an anchor broadcast received by a set of nodes $R \subset V$. Let $S \subseteq R$ be the set of nodes that actually change their next links as a result of executing Algorithm 1 Since proper handoffs are assumed, $\left\{r, r^{\prime}\right\} \subseteq S$. Algorithm 1 dictates that all nodes in $S$ points to $r^{\prime}$ (with the exception of $r^{\prime}$ which points to the MB) after the update. That is, the next links of nodes in $S$ form a routing tree rooted at $r^{\prime}$.

Let $T(r)$ be a spanning routing tree of $G$ rooted at node $r$, and $F_{S}$ be the forest obtained by removing the next links of nodes in $S$ from $T(r)$. Since $r \in S$, each tree in $F_{S}$ is rooted at a node in $s \in S$. By definition, none of the edges in any tree $T_{s} \in F_{S}$ is changed. Since next links in $S$ forms a routing tree rooted at $r^{\prime}$, next links in $F_{S}$ and $S$ form a spanning routing tree rooted at $r^{\prime}$.

While message losses are common in WSN environments, most message losses do not effect the correctness of DTR (Theorem 1), as the definition of proper handoffs only require reliable message delivery between the $\mathrm{MB}$ and the old and new anchors. For the remaining nodes, message loss is only a nuisance, rather than constituting a correctness problem. Message losses at these nodes may result only in degraded performance, since their path is not updated to point to the new anchor in the most direct/shortest manner. But, since the previous routes point to the old anchor, which points to the new anchor, due to Theorem 1 the network is still routingconnected.

The routing-connected network property is violated only when the old or new anchor miss an anchor broadcast. DTR deals with this problem in two timescales: short and long terms. In the short term the impact of message losses are reduced through message redundancy. Increasing the anchor broadcast frequency at the MB improves the chances that all neighbors receive the information about the new anchor node. When this scheme is insufficient, there may be partitions in the network due to improper handoffs. In the long term, since the $\mathrm{MB}$ is mobile, MB is very likely to move over the partitioned regions eventually. This will, in turn, fix the problem and enable the buffered packets to be relayed to the MB.

\section{Handoff Connectivity}

The correctness of DTR depends on the success of handoffs, which is in turn imposed by the geometry and topology of the network. Here, we focus on planar deployments and capture these required geometric and topological properties.

In data spider, MB invariantly maintains its closest node as the anchor node. A useful abstraction for capturing this property is the Voronoi diagram of WSN nodes based on their geographical locations. When the MB is in one of the Voronoi cells, its closest node, by definition, is the WSN node corresponding to that Voronoi cell. Thus, as long as MB stays in that Voronoi cell, the anchor node is unchanged.

With this anchor node definition, we identify the requirements for having proper handoffs as follows. Let $P$ denote a point in the deployment area and $V_{P}$ be the set of nodes which are closest to $P$. So, if $P$ falls inside a Voronoi cell, then $V_{P}$ consists of a single node, the WSN node corresponding to that Voronoi cell. If $P$ falls on a Voronoi cell boundary, then $V_{P}$ consists of the neighboring (i.e., adjacent) nodes for this Voronoi cell boundary. 


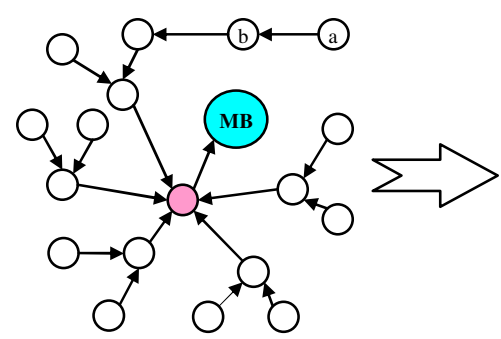

(a) Before Broadcast

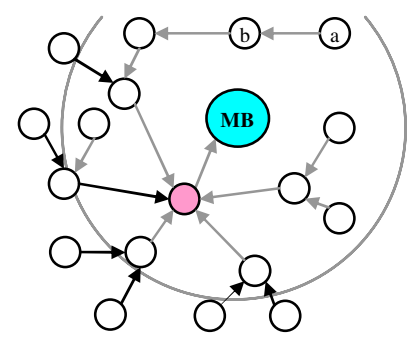

(b) Broadcast

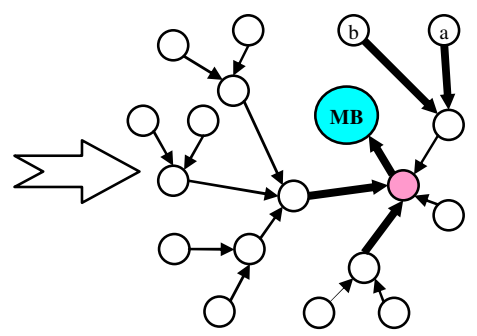

(c) After Broadcast

Fig. 1. Demonstration of DTR as MB moves from one anchor to another. The touched edges are gray edges in (b) and actual changes are bold edges in (c).

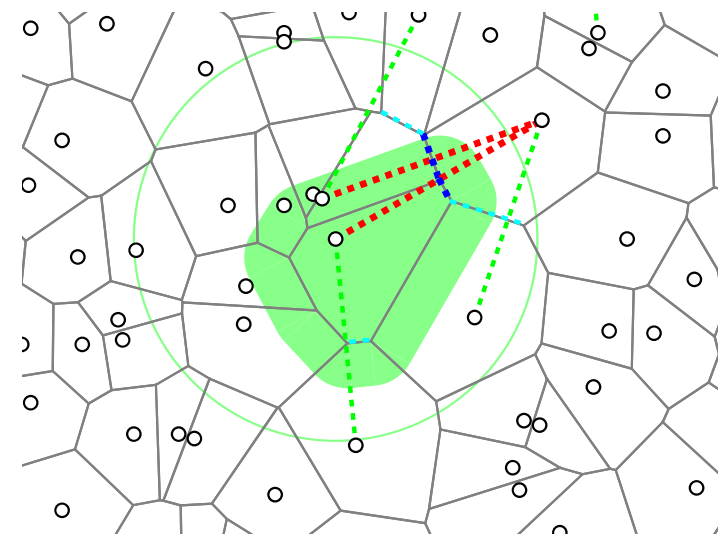

Fig. 2. The interaction of Voronoi cells and communication requirements. Shaded region shows possible locations of MB at next update when it starts with anchor node in the center of shaded region. Circle around the shaded region is the approximated reliable communication range. Green(light) dashed lines correspond to neighbors where direct handoff is not possible. Red(dark) dashed lines correspond to neighbors where no handoff is possible.

We call a WSN deployment handoff connected when all points $P$ in deployment region satisfy:

1) For all nodes $n \in V_{P}, n$ can reliably communicate with a node placed at $P$.

2) For all nodes $n, m \in V_{P}, n$ and $m$ can reliably communicate with each other.

In other words, in a handoff connected network (1) the MB sitting on a Voronoi cell boundary can communicate with the nodes in the adjacent Voronoi cells, and (2) any pair of Voronoi neighbors can communicate with each other.

The above handoff connectivity definition is valid when the updates of the MB are continuous. Since we use discrete/periodic anchor broadcasts, we extend this definition for our model. Let $\lambda=v_{B S} * T_{\text {update }}$ be the maximum distance the MB can travel between two location updates. We now require the anchor node to be able to receive messages from the MB when it is at most $\lambda$ away from the Voronoi cell. Moreover, for proper handoff, any cell that falls to this region should be in communication range. Figure 2 demonstrates this requirement.

To generalize the handoff region we extend the set of nearest nodes $V_{P} . V_{P}^{\lambda}$ to be the set of nodes which are at most $\lambda+d_{\min }$ away from point $P$ where $d_{\text {min }}$ is the minimum distance to any node in network from $P$. Thus, using $V_{P}^{\lambda}$ we generalize handoff connectivity as follows:
A WSN deployment is said to be $\lambda$-general handoff connected when all points $P$ satisfy:

1) For all nodes $n \in V_{P}^{\lambda}$, $n$ can reliably communicate with a node place at $P$.

2) For all nodes $n, m \in V_{P}^{\lambda}, n$ and $m$ can reliably communicate with each other.

\section{Extensions to DTR}

Handoff connectivity addresses only the immediate neighborhood of the anchor node. Broadcasts on the other hand can be made stronger with better transmitters on the MB. Moreover, even WSN nodes can receive broadcasts from nonneighboring Voronoi cells. These receptions can be utilized to improve the performance of DTR as follows.

For this operation, nodes depend on neighborhood information about their neighbors. That is, nodes share neighborhood information with their neighbors, so that they can create twohop routes to the anchor node when singlehop routes are not possible. If the anchor is not an immediate neighbor of the node, the node chooses its neighbor which is an immediate neighbor of the anchor. In case there are multiple neighbors satisfying this condition, the closest one to the anchor is chosen as the next node. As long as the chosen intermediate nodes also received the anchor broadcast this operation extends the handoff connectivity. We call this operation indirect handoff. We show neighbor nodes where only indirect handoff is possible with green(light) edges in Figure 2 Non-anchor nodes also benefit from our indirect handoff extension, as is the case for nodes $a$ and $b$ in Figure 1.

\section{MB ALGORITHM}

The MB algorithm complements synergizes with the DTR algorithm to achieve efficient data collection. We present the basic MB algorithm in Algorithm 2 MB ensures two things:

(1) The MB broadcasts an anchor message announcing the closest node to itself periodically. This enables DTR to track MB correctly and update the tree accordingly so DTR can keep delivering data packets to the MB. In order to detect and announce anchor changes properly, the MB should determine the closest node to itself. To this end, we require that MB knows its location as well as the locations of nodes in the network. This is achievable by equipping the MB with a GPS and the coordinates of the WSN nodes. Having a GPS on the MB is relatively cheap, and the MB can also utilize its GPS 


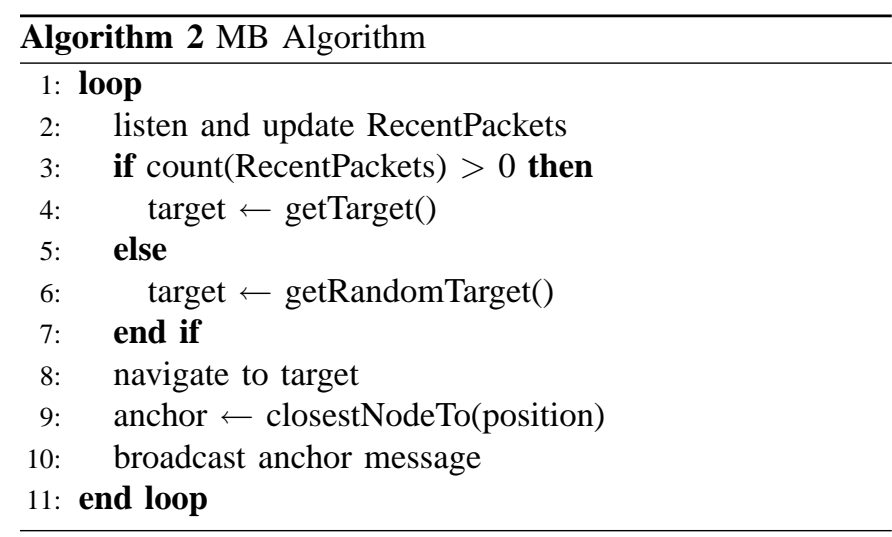

to locate and collect the nodes after deployment. (Alternative localization techniques based on Kalman filtering and RSSIbased determination of an approximately-closest node may also be considered.)

(2) The MB relocates to follow data generation in a besteffort manner. This relocation enables the effective length of data relaying paths in DTR to be only a couple of hops, improving both the reliability and the lifetime of the data collection. In order to track the data generation in the network, MB utilizes the recent data packets that DTR routes to itself to decide where to move to next. MB defaults to a random walk when there are no packets since this might indicate a disconnection of the network. In such a case, random walk may help the MB to repair the partitioning and re-establish a connected network where DTR can start delivering the data generated to the MB. Otherwise, MB uses the getTarget() function to decide how to relocate based on the recently received packets. We propose two heuristics for this function:

trailSource. Here, the MB inspects the source field of the data packets and sets the relocation target to be the source of the packet generation (median of the source locations). Although it seems like this is a direct approach and should not lead to much problems, we show in Section IV] that when the network is not regular (has holes in it) trailSource leads to many improper handoffs and suffers severe performance penalties.

trailFlow. Here, the MB tries to go to the center of packet flow. In contrast to trailSource that calculates the center of data generation, trailFlow calculates the center of data forwarding from the singlehop neighbors of the MB. Since packet forwarding is done over reliable edges, trailFlow directs the MB to avoid the holes in the network implicitly (as a side benefit), so even in irregular and sparse networks trailFlow ensures successful handoffs. Our simulation results in Section IV] show that trailFlow consistently performs the best compared to the other heuristics.

\section{Simulation}

\section{A. Setup}

Simulator. We built our simulator on top of the JProwler simulator [12] and implemented support for mobility for JProwler. Our implementation is heavily optimized towards

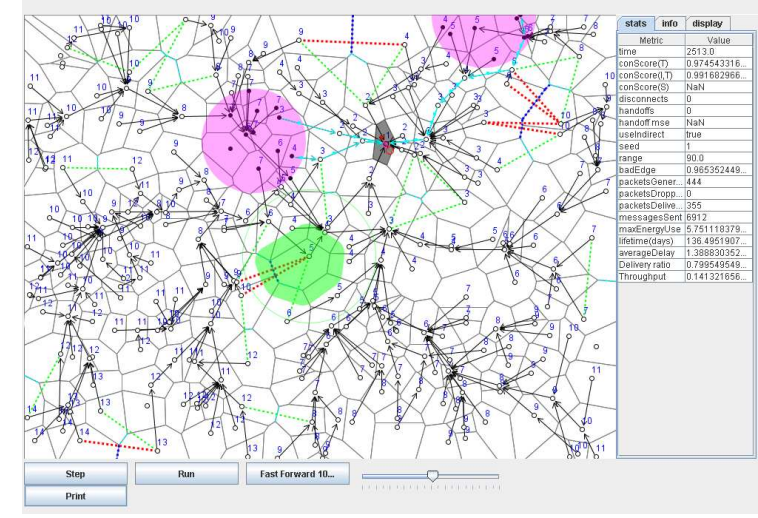

Fig. 3. A screenshot of the simulator

large number of static nodes and a small number of mobile nodes. In our simulator, we also updated the radio model of JProwler to reflect the mobility of the mobile nodes, while keeping the static nodes unchanged.

In order to address energy efficiency questions we keep track of energy use in our simulation. Our simulator uses CSMA with BMAC low-power-listening [10] for the MAC layer and the associated energy model to calculate the energy used in each node. In our simulation we obtain fine grain information about packet arrivals and noise and replace the approximate values used in [10] with these values to better capture the energy use in each sensor node. We ignore the energy cost of computation, but since our algorithms are relatively simple we argue that the energy cost of the computation specific to algorithm is negligible.

Our simulator is parametrized extensively, so it is suitable for modeling and investigating other MB algorithms quickly. Figure 3 shows a screenshot of our simulator. Our simulator is available at http://www.cse.buffalo.edu/ubicomp/dataSpider/.

Simulation setup. We ran each set of simulations for 72 simulation hours. Each simulation includes an initial neighborhood discovery and initial flooding phase. Neighborhood discovery phase reduces the disconnections and message losses as reliable links are identified and each node discovers its neighbors. This neighborhood information is later utilized in performing indirect handoffs.

As in [9], we model the data generation activity in the environment with a moving disc to denote the ROI. All WSN nodes covered by this disc generate data with a predetermined rate. The nodes then try to forward this data to the MB if they have a valid next link. A node buffers data if the channel is busy, or if it does not have a valid next link-which may happen after an improper handoff. We rely on work in [13] to generate realistic human/animal like mobility patterns for the ROI. Our implementation of the model is faithful to the model with minor modifications to scale the deployment region.

We set the simulation area as a $160 \mathrm{~m}$ by $120 \mathrm{~m}$ rectangular region. Unless otherwise stated we work with 300 mica 2 nodes in this region. We constrain the $\mathrm{MB}$ to this region and assume that there are no significant obstacles to obstruct mobility within the region. Our mobility simulation is intentionally 
kept simple. We do not model problems in relocation such as mechanics of maneuvering, localization, and obstacle avoidance in order to concentrate on the networking aspects of the problem.

Protocols we compare with. We are primarily interested in evaluating the data spider system which consists of DTR and the MB algorithms, trailSource and trailFlow, described in Sections II and III. For comparison, we also consider three other protocols, namely, static, random, and salmon.

In the static protocol, the basestation is static and is located in the center of the network. The data is routed to the based using a convergecast tree rooted at the basestation. As we discussed in the Introduction this scheme is prone to hotspots around the basestation, and also results in long multihop paths for data relaying.

The random protocol is similar to trailSource and trailFlow in that it also uses the DTR protocol to reconfigure the data collection tree as the MB relocates. However, as for the relocation algorithm, instead of trying to follow the data generation, the random protocol prescribes relocating the $\mathrm{MB}$ to a random location all the time. While this protocol avoids the hotspot issue (since it uses an MB and DTR), it is prone to long multihop paths for data relaying as it does not follow the data generation.

Salmon protocol uses the same MB algorithm we used in our previous work, data salmon [9]. Salmon does not use DTR and constrains the relocation of the $\mathrm{MB}$ to occur only along the edges of the existing tree. In other words, the existing tree is not modified, except for the relocation of the root of the tree from one node to one of the neighboring nodes (which is achieved by flipping the direction of the edge between these two nodes). In this scheme, the MB chooses the neighbor that forwards the majority of the traffic to relocate to. As our simulation results exhibit, this scheme has problems with reliability (since only one edge is modified, this constitutes a risk of single point of failure) and cannot follow the data generation successfully (since the MB relocation is restricted to the existing tree structure, MB needs take long detours when the ROI leaves the current subtree for another subtree).

Metrics. We concentrate on three metrics to measure performance of the system. The latency metric measures the average delay in packet deliveries, from their generation time to their arrival to MB. The second metric, packet delivery rate, is the ratio of the delivered packets to $\mathrm{MB}$ versus the number of packets generated. The final metric is the estimated lifetime of the network. We define the lifetime to be the time passed until the first node failure due to battery depletion in the network. By utilizing the fine-grained energy-use information from our simulation and the total energy stored in standard AA batteries, we arrive to our estimated lifetime figures.

\section{B. Results}

Here we present our simulation results for the metrics described above under the following parameter categories.

Node density. We first investigate the effect of node density on the performance. As the number of nodes increase, since the distance between anchor nodes would be decreasing, we expect better connectivity of the network and reduced number of improper handoffs. Increased density also corresponds to increased data rates and more contention reducing the lifetime of the network. Figure 4 presents this axis of the investigation. We observe very high latencies when node density is low. This is due to frequent disconnections. Packets are buffered when handoffs can not be completed successfully and they are later retrieved on an opportunistic basis, but this results in high average latencies. Data spider heuristics trailFlow and trailSource consistently outperform other protocols with respect to packet deliveries and network lifetime. An interesting result of this experiment is to show that even random mobility leads to better delivery ratios than the static when the density is critically low. Random mobility leads to worse delivery ratios when the density increases, yet it still leads to longer lifetimes than static.

Indirect handoff. Here we try to quantify the performance improvement due the indirect handoff extension. We test this through experiments with trailFlow. Indirect handoff provides better average latencies, and up-to $5 \%$ improvement in packet delivery rates. The benefit of indirect handoff is most significant in expected lifetime which is improved by $20 \%$.

Speed of region of interest (ROI). The ability to track ROI is a significant advantage for data spider, but the performance of tracking is affected by the speed of ROI. In our experiments depicted in Figure 6 we investigate the effect of speed of ROI to the performance. Since we use a fixed speed for MB, increasing the speed of ROI makes tracking the data more difficult. As expected static and random heuristics are not effected by the ROI speed. We observe significant increase in average delay in trailSource heuristic. This increase is related to increased number of bad handoffs, which leads to partitions of network. trailFlow avoids this problem as packets follow the network topology and the MB follows the packets. Even with increased ROI speed, data spider algorithm improves the lifetime of network up to 3 times over static basestation.

Number of ROI. We next consider the effects of increasing number of ROIs on the performance. As these ROIs move independently from each other, the optimal location of $\mathrm{MB}$ would vary significantly and the static MB starts to become a better alternative. Our simulation results are shown in Figure 7 We observe the effect of disconnections in trailSource heuristic in this experiment as well. The difference between data delivery rates decrease as data spider heuristics can not follow all the ROIs at the same time. Lifetime of the network is also inversely affected as the $\mathrm{MB}$ is constrained to a smaller region trying to follow all ROIs simultaneously. With 4 ROIS, the performance of data spider is similar to random $\mathrm{MB}$ in terms of network lifetime, which is still more than $100 \%$ improvement over the static basestation.

Locality of data generation. Locality of data generation is important for the performance of data spider. In the previous set of experiments we explored this a little by increasing the number of ROIs. Here we quantify data locality by removing the dependency of data generation to the ROI. In this setup, 

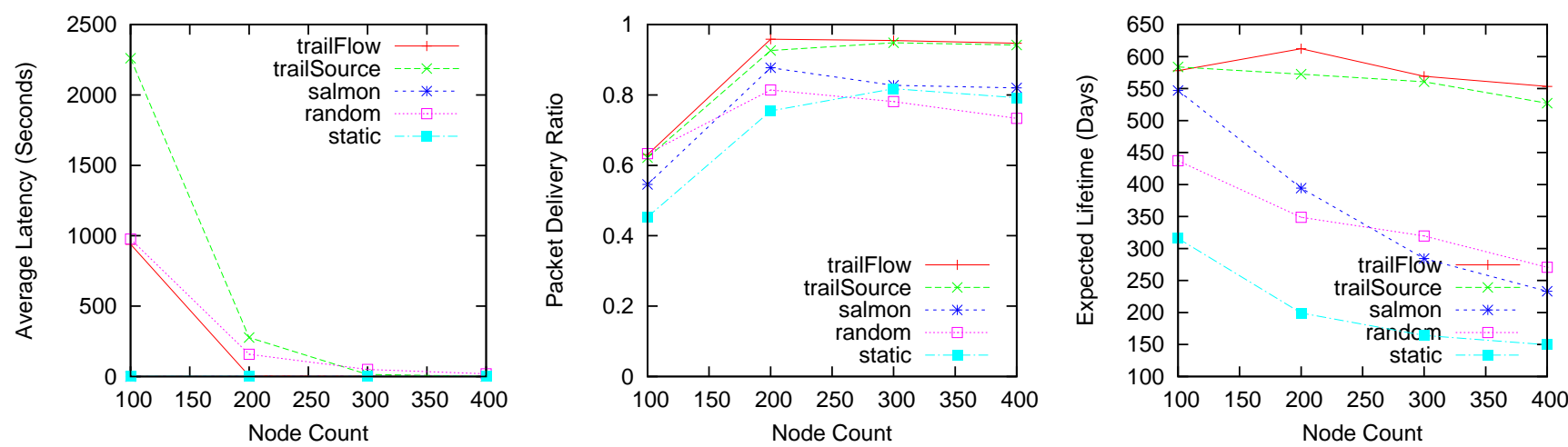

Fig. 4. Effect of number of nodes on performance
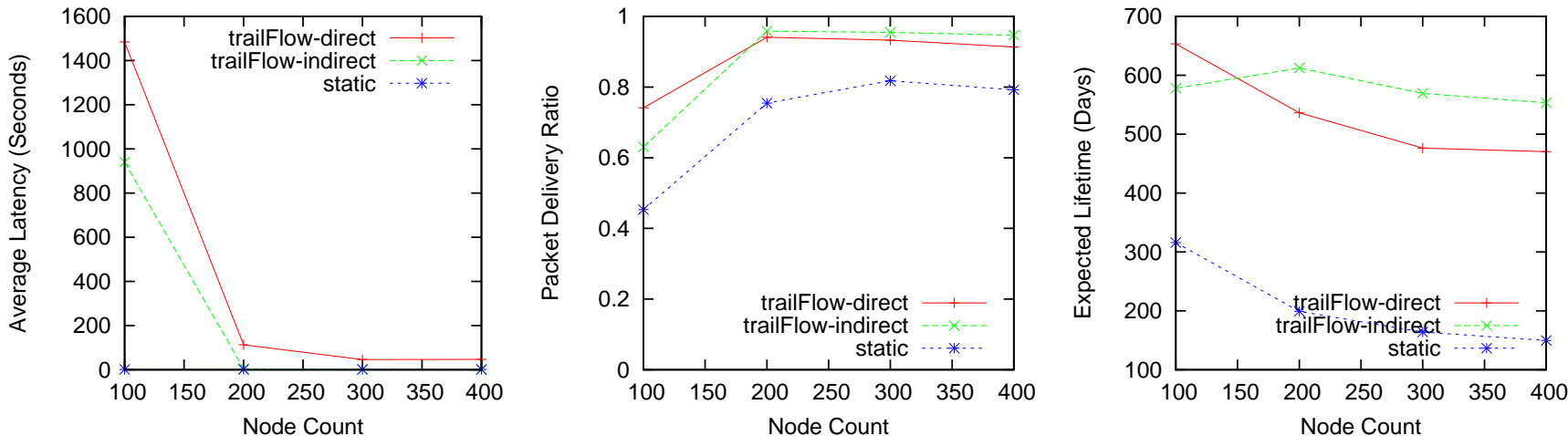

Fig. 5. Effect of indirect handoff on performance
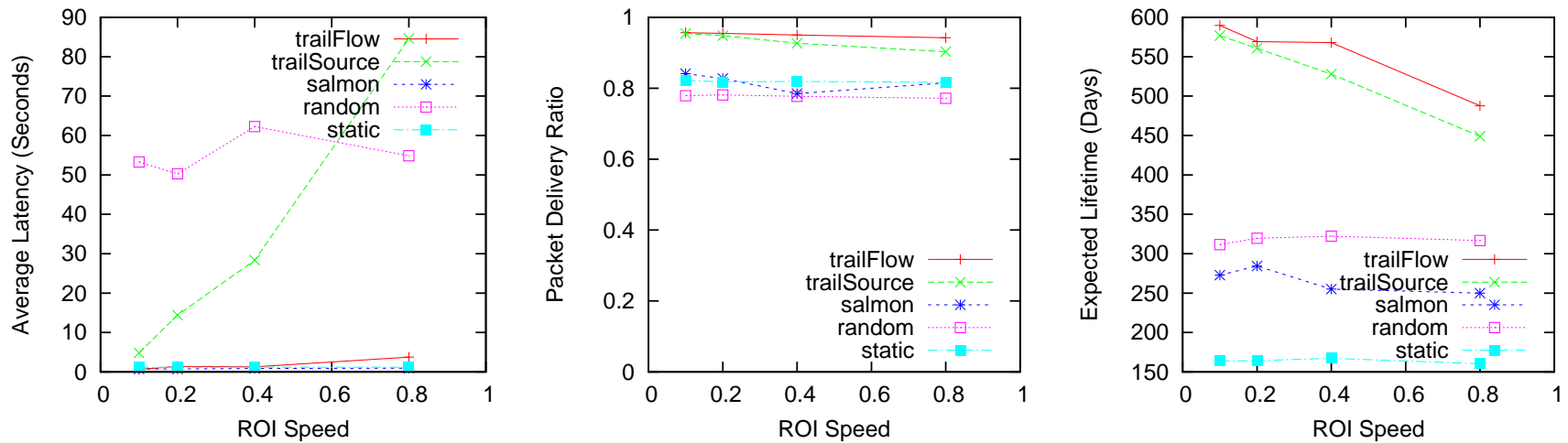

Fig. 6. Effect of speed of region of interest on performance
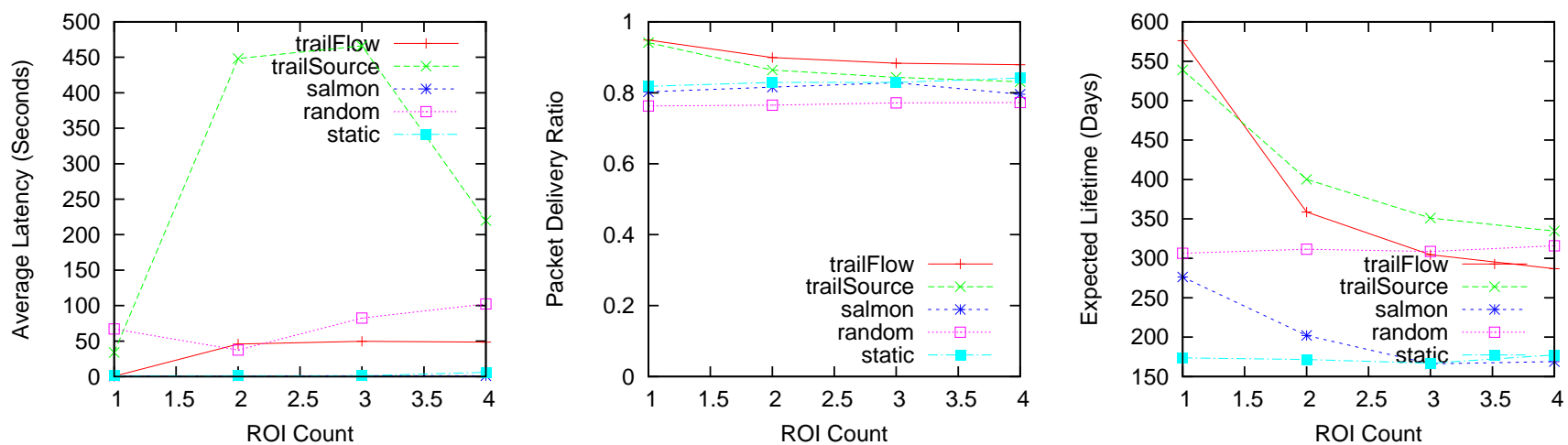

Fig. 7. Effect of number of region of interests on performance 
each node generates a random number and if this number is less than the data locality parameter, the packet is generated in the ROI, otherwise the packet is generated in a random node chosen uniformly over the network. Thus, a data locality of 1 corresponds to the regular data generation and a data locality of 0 corresponds to totally uncorrelated (uniformly random) data generation. Figure 8 summarizes the results of this investigation. In general we observe that decreased locality leads to decreased performance for data spider in all three metrics. Even when one fifth of the data is generated within the ROI, data spider tends to follow this correlated portion of data generation, which results in reduced performance as the rest of the data is generated uniformly. In a sense, this kind of data generation tricks MB to suboptimal locations. One of the most interesting results of this experiment is the relatively high performance when there is no correlation in data. This result stems from the reduced congestion in the network when data is generated uniformly in the network. In that case, MB stays close to the center of the area as data generation is uniform, but lifetime and delivery ratios are still better than static basestation, as in data spider the MB can wander around leading to better load balancing.

Frequency of anchor broadcasts. An important trade off in data spider is the choice of anchor broadcast frequency. Increasing the number of anchor broadcasts allows for sparser deployments but also results in contention with other traffic. We investigate the effect of MB anchor broadcasts in Figure 9. Average delay consistently improves with increased number of anchor broadcasts but other metrics show an optimal value around $0.01 \mathrm{hz}$ which is the value used in all other experiments.

Multiple MBs. Figure 7 showed that increasing the number of ROIs reduced the ability of data spider to track them. Here we show how the increased number of ROIs are better handled with multiple MBs. We test the performance of data spider with multiple MBs in Figure 10. As we mentioned in the Introduction, data spider extends readily to allow multiple MBs to share the same network without any need to change the DTR or MB algorithms. In this experiment neither the network nor the MBs are aware of the multiple MBs. However, we still observe an emergent cooperation and division of labor leading to improved performance. MBs partition the network since each node only has one next node, moreover these partitions dynamically change over time due to MB broadcasts. Even if all MBs converge to same anchor, the competition for data allows MBs to diverge and cover different ROIs. We obtained these very promising results with data spider despite lack of explicit coordination. An interesting research question is how to coordinate $\mathrm{MBs}$ in a cooperative manner to improve performance even further.

\section{CONCLUding Remarks}

We presented an efficient holistic MB-based data collection system, data spider, which consists of two subsystems. The WSN component of data spider is a very lightweight dynamic tree reconfiguration protocol, DTR, which follows the location of the MB and re-weaves/mends the original tree locally to maintain an always connected network rooted at the MB. The MB component of data spider relies on the data delivered to it by DTR and complements DTR by trailing towards the data generation. This, in turn, reduces the number of hops data needs to be relayed to the MB and boosts the reliability and lifetime of DTR. While both protocols are very simple and lightweight, combined they lead to significant improvements in the reliability and lifetime of data collection, especially for monitoring applications with highly spatiotemporal data generation.

We provided extensive simulation results where we evaluated the latency, cost, and network lifetime metrics of the data spider system under a wide number of varying parameters, and compared and contrasted data spider with other systems. We also presented analytical results about data spider and formulated the handoff connectivity requirements needed for performing a proper handoff of the MB.

Although we focused on the data collection problem, our data spider framework readily applies also to the pursuerevader tracking problem [14] by treating the ROI as the evader and the $\mathrm{MB}$ as the pursuer. Our experiments here showed that the trail-flow algorithm for the MB managed to implicitly route the $\mathrm{MB}$ around the holes in the network. In future work we will investigate other algorithms that may result in similar desirable properties for tracking. Our experiments also showed that, in the data spider system, multiple MBs coexisted nicely on the same network to trail multiple ROIs without any explicit coordination or cooperation. In future work we will investigate coordination and cooperation mechanisms of multiple MBs for more efficient evader tracking.

\section{REFERENCES}

[1] M. Batalin, M. Rahimi, Y. Yu, D. Liu, A. Kansal, G. Sukhatme, W. Kaiser, M. Hansen, G. Pottie, M. Srivastava, and D. Estrin, "Call and response: experiments in sampling the environment," in SenSys '04: Proceedings of the 2nd international conference on Embedded networked sensor systems, 2004, pp. 25-38.

[2] R. Szewczyk, A. Mainwaring, J. Polastre, and D. Culler, "An analysis of a large scale habitat monitoring application," In Proceedings of the Second ACM Conference on Embedded Networked Sensor Systems (SenSys), 2004.

[3] A. Arora, P. Dutta, S. Bapat, V. Kulathumani, H. Zhang, V. Naik, V. Mittal, H. Cao, M. Demirbas, M. Gouda, Y.-R. Choi, T. Herman, S. S. Kulkarni, U. Arumugam, M. Nesterenko, A. Vora, and M. Miyashita, "A line in the sand: A wireless sensor network for target detection, classification, and tracking," Computer Networks (Elsevier), vol. 46, no. 5, pp. 605-634, 2004

[4] A. Arora and et. al., "Exscal: Elements of an extreme scale wireless sensor network," 11th IEEE International Conference on Embedded and Real-Time Computing Systems and Applications, 2005.

[5] R. C. Shah, S. Roy, S. Jain, and W. Brunette, "Data mules: modeling a three-tier architecture for sparse sensor networks," Proceedings of the First IEEE International Workshop on Sensor Network Protocols and Applications, pp. 30-41, 2003.

[6] A. Somasundara, A. Ramamoorthy, and M. Srivastava, "Mobile element scheduling for efficient data collection in wireless sensor networks with dynamic deadlines," in RTSS '04: Proceedings of the 25th IEEE International Real-Time Systems Symposium (RTSS'04), 2004, pp. 296305.

[7] Y. Gu, D. Bozdag, E. Ekici, F. Ozguner, and C. Lee, "Partitioning based mobile element scheduling in wireless sensor networks," IEEE SECON, pp. 386-395, 2005. 

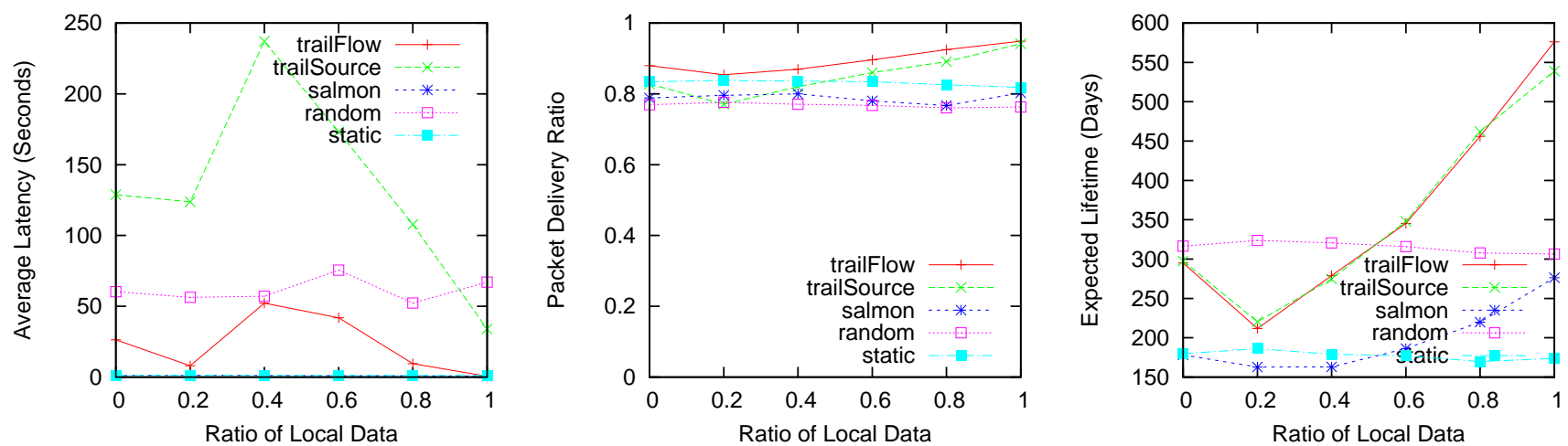

Fig. 8. Effect of data locality on performance.
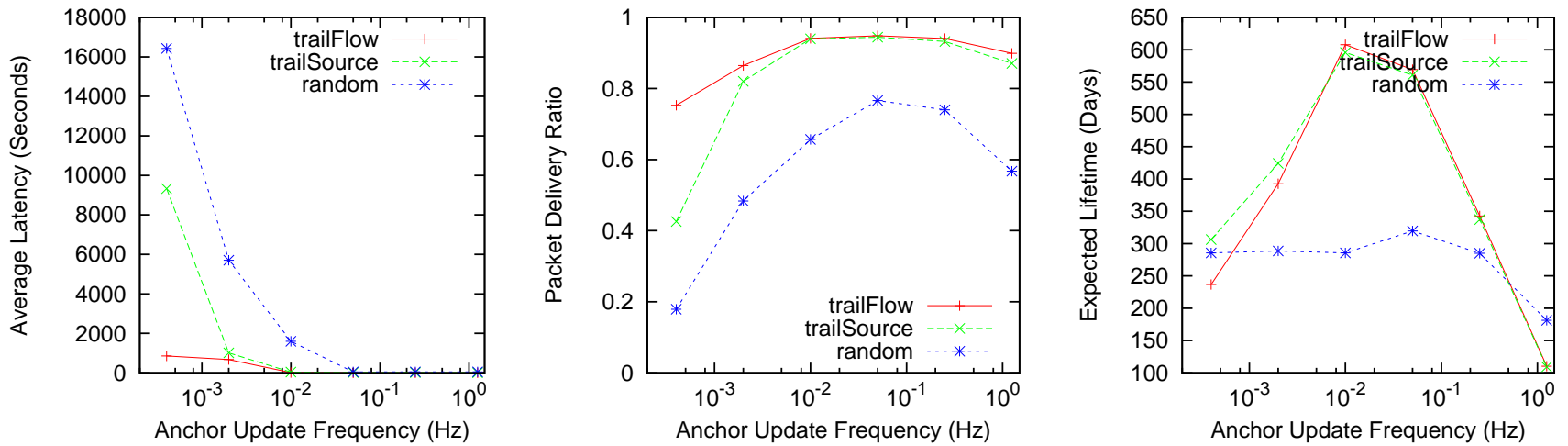

Fig. 9. Effect of $\mathrm{MB}$ anchor broadcasts on performance.
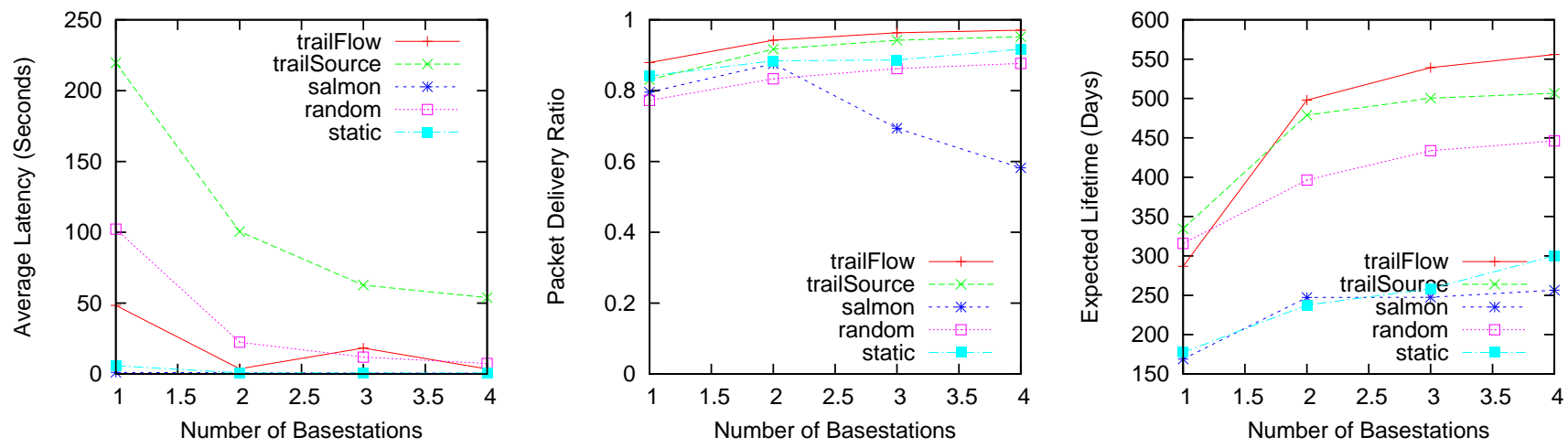

Fig. 10. Effect of number of MBs on performance with 4 independent ROIs.

[8] S. Basagni, A. Carosi, E. Melachrinoudis, C. Petrioli, and Z. Wang, "A new milp formulation and distributed protocols for wireless sensor networks lifetime maximization," in Communications, 2006. ICC '06. IEEE International Conference on, vol. 8, June 2006, pp. 3517-3524.

[9] M. Demirbas, O. Soysal, and A. S. Tosun, "Data salmon: A greedy mobile basestation protocol for efficient data collection in wireless sensor networks," IEEE International Conference on Distributed Computing in Sensor Systems (DCOSS), pp. 267-280, 2007.

[10] J. Polastre, J. Hill, and D. Culler, "Versatile low power media access for wireless sensor networks," in SenSys '04: Proceedings of the 2nd international conference on Embedded networked sensor systems, 2004, pp. 95-107.

[11] W. Zhang and G. Cao, "Dctc: Dynamic convoy tree-based collaboration for target tracking in sensor networks," IEEE Transactions on Wireless Communication, vol. 3, no. 5, pp. 1689-1701, 2004.

[12] G. Simon, P. Volgyesi, M. Maroti, and A. Ledeczi, "Simulation-based optimization of communication protocols for large-scale wireless sensor networks," IEEE Aerospace Conference, pp. 255-267, March 2003.

[13] W. jen Hsu, T. Spyropoulos, K. Psounis, and A. Helmy, "Modeling timevariant user mobility in wireless mobile networks," in INFOCOM 2007. 26th IEEE International Conference on Computer Communications. IEEE, May 2007, pp. 758-766.

[14] M. Demirbas, A. Arora, and M. Gouda, "A pursuer-evader game for sensor networks," Proceedings of the Sixth Symposium on SelfStabilizing Systems(SSS'03), pp. 1-16, June 2003. 